\title{
Examining the Morphological and Physiological Comparisons of OVX Murine Bone vs. Mini Pigs, Rats, and Humans
}

\author{
Shania Jafri ${ }^{1}$, Karrington Hendrix ${ }^{1}$, Pedro Cuevas ${ }^{1}$, Igor Pilawski ${ }^{2 \#}$, and Jill Helms ${ }^{1 \#}$ \\ ${ }^{1}$ Stanford University, Palo Alto, CA, USA \\ ${ }^{2}$ Vétérinaires sans Frontìeres Germany, Berlin (Formerly: Stanford University, School of Medicine, \\ Department of Surgery) \\ \#Advisor
}

\section{ABSTRACT}

Osteons are the structure and the base foundation of the human skeletal system. This cylindrical structure contains blood vessels and nerves that supply the bone matrix in humans. To study bone remodeling and bone diseases, mini pigs and rats have mainly been effective models for humans. This study was conducted to examine mouse bone structures in comparison to human, mini pig, and rat bone structures. This addresses the Three Rs principle of clinical testing on animals and proves that mice should be used as models for humans instead of mini pigs and rats. Many scientists prefer not to use mice as models for studying human bone diseases because it has been suggested that their skeletal systems are morphologically and physiologically different-as seen through aging effects. Because mice are easier to produce and can grow at a faster rate, they are more cost and time efficient to use in labs compared to rats and mini pigs. Aniline blue, Ploton silver, picrosirius red stains and TRAP and ALP cellular assays were conducted to analyze bone structures to compare with humans, mini pigs, and rats. Our data did support the hypothesis as explicit similarities between mice bone and other samples such as rats, mini pig, and human was deduced. This study concluded that there were little limitations present by using murine bone as samples for human when studying bone diseases.

\section{Introduction}

A common subject that is used for laboratory testing are mice, which allow scientists to study single variables to compare to humans. When discussing animal models, however, review papers discourage scientists from using mice in bone research since their bone morphology is vastly different (Jilka, 2013). For instance, because mice bone density is small, scientists would rather use animals with a larger bone density such as mini pigs or rats as models for humans. With bone degenerative diseases affecting an estimated 200 million people worldwide mice are most accommodable for biology research (Sözen, 2017).

\section{Osteons}

An essential bone structure called osteons play a major function in bone development and remodeling. Osteons are also a common structure that has prompted differences between scientists when regarding the similarities between human and murine bone morphology. Osteons are bone in a constant state of turn over. At the center of each osteon is the Haversian canal, which contains capillaries and nerve fibers that allow the bone to receive oxygen and nutrients 


\section{Journal of Student Research}

(Shahar el al., 2011). Osteons are formed from osteoclasts burrowing in the bone to form the Haversian canals (Parfitt 1994). Osteoclasts resorb aged bone and osteoblasts build a new bone matrix. The synchronized action of osteoblasts and osteoclasts define bone remodeling (Fowler et al., 2017). Any bone can be reformed young or old as long as it is weight bearing. The osteocyte is the orchestrator that singles the systems to telegraph information through the canaliculi (Schaffler et al., 2012). The canaliculi rotate nutrients to make the osteon stable and discards waste products from the osteocytes.

By locating these very systems and components within murine bone, it will address the idea that humans and mice are morphologically similar, allowing mice to be used more often than mini pigs and rats. Other structural elements of osteonal bone include lacunae and cement lines. The outer border of an osteon is marked by cement lines, a region of collagen-poor bone matrix indicating resorptive activity and bone remodeling. Cement lines represent weak and fracture-prone areas of the bone and are a result of the formation of secondary osteons (Skedros et al., 2005).

\section{Cortical and Trabecular Bone}

Trabecular bone-also known as spongy bone-has a significantly lower density than cortical bone. Within the bony labyrinth that is trabecular bone, bone marrow that contains blood, stem cells, nerves are housed within. It is important to note that there is no bone marrow in cortical bone because it is very dense. Cortical bone, found in the outer layers of bone, can form osteons, an essential structure scientist examines for bone diseases. This is the reason long bone, specifically femoral bone, was used in aniline blue due to the likelihood of finding osteons. Ploton silver was used to identify osteons and bone remodeling through examining the configurations of the lacuno-canaliculiar systems. Other cellular assays such as picrosirius stain, TRAP, and ALP were also used to examine bone remodeling and physiology in trabecular bone.

In Pilawski's paper, it was stated that trabecular bone is structurally and physiologically is similar across all species (Pilawski et al., 2020). Pilawski and colleagues also (2020) compared the bone volume of mini pig, mice, and rats found that the bone mineral density was equal. It was also concluded that collagen maturation and bone remodeling was conserved amongst all three species.

\section{Bone Tissue Samples}

Longitude bone was the most effective to examine remodeling such as femoral bone because it is constantly under dynamic stress (Pereira, 2015). The shaft area of femoral bone was examined in this experiment because it is already known that osteons cannot be found in trabecular bone. This would eliminate suspicions of osteon-like structures appearing within trabecular bone. By examining mice femur, there is a higher probability to observe bone remodeling and osteons which would make mice femur morphologically and physiologically similar to humans. Demonstrating the existence of osteons in murine cortical bone can disprove some of the aforementioned criticism for mouse animal models. The relaxation and reloading processes are conducted by the lacunar-canalicular system. Consequently, if there are lacunae then there are osteons even though murine "lacks osteonal remodeling of cortical bone" (Jilka, 2013).

Alveolar bone is craniofacial tissue that has both forms of bone: an outside cortical bone layer and inside trabecular bone layer. This bone was used to examine bone remodeling and physiology because trabecular bone turnover rate is higher than cortical bone (Ott, 2018).

\section{OVX (Ovariectomized)}

Ovariectomized mice are the best used for clinical studies of bone diseases and postmenopausal bone lose in adult women. Because OVX mice have their ovaries removed they experience an estrogen deviancy which can mimic such 


\section{Tournal of Student Research}

conditions (Inada, 2011). By being able to observe the beginning stages of development there is a greater likelihood of observing osteons.

\section{Ethics}

There are more well-established protocols and functioning antibodies for mice than mini pigs and rats. For instance, a common human protein RUNX2 and RANKL, two essential regulators in bone physiology, functioned properly in mice but were unable to work in mini pig (Pilawski et al., 2020). This was because mini pig tissue is more difficult to process. Since mice have been commonly used in research in general, there is more information and familiarity on them. This parallels the Three R's Principle of reduction, refinement, and replacement practices the minimal use of animals and using strategies that minimize as much pain as possible (Crespi-Abril, 2021). This very principle stresses the importance of choosing the appropriate animal model for experimentation for bone diseases (Crespi-Abril, 2021). Current animal models such as pigs can take up to two years for their bones to mature and later be utilized for experimentation. During those two years, resources and time are wasted, especially for patients who suffer from bone diseases and await a cure. There is a critical need for new animal models who match the appropriate qualifications such as age, measurements, and bone maturity and because most of laboratory protocols are geared towards mice, mice are the most viable models for human bone diseases (Turner et al., 2001).

This study questions the contrary statements that mice cannot be used for bone disease research by examining tracings of osteoblasts and osteoclasts in mice. We hypothesize that osteonal remodeling occurs in mature mouse bone which would show that mice are morphologically and physiologically similar to humans compared to any other animal such as mini pigs and rats. This would address the fact that mice are more compatible models-ethically, morphologically, and physiologically-when studying adult bone diseases in humans.

\section{Material and Methods}

\section{Tissue Preparation}

All procedures followed protocols approved by the Stanford Committee on Animal Research. OVX mice were purchased from Charles River Laboratories Inc, MA, USA and housed in a temperature-controlled environment with 12-h light/dark cycles. Two-month-old female OVX mice were sacrificed, and their femurs were examined. The skin and outer layers of muscle were removed, and tissues were fixed in $4 \%$ paraformaldehyde overnight at $4{ }^{\circ} \mathrm{C}$. The samples were decalcified in a heat-controlled microwave in 19\% EDTA for 2 weeks. After demineralization, specimens were dehydrated through an ascending ethanol series prior to paraffin embedding. Eight-micron-thick longitudinal sections were cut and collected on Superfrost-plus slides for histology. The following stains were then conducted on the slides: histology of aniline blue, Ploton silver staining, and picrosirius red; cellular assays including TRAP (tartrate-resistant acid phosphatase) and ALP (alkaline phosphatase).

OVX mice are commonly used to study bone loss and bone remodeling and contain a significantly lower amount of estrogen. Conducting the experiment, different staining processes were used to examine murine femur bone in order to find the presence of osteons at two-month-old. Samples were examined through light microscopy; oil immersion method was used to increase the resolving power of the microscope to see samples at 60x objection.

Alveolar bone samples from patients were collected as part of a multicenter clinical investigation (Chen et al. 2018). Procedures and materials were approved by local ethics committees, Institutional Research Boards (WIRB\#20160967 for US private practices), and all conformed to STROBE guidelines and followed principles of the Declaration of 


\section{Journal of Student Research}

Helsinki. For histological and immunohistochemical analyses, tissues from 3 male and 2 female healthy volunteers, ranging in age from 59-76, were included in this study.

Alveolar bone samples from 11 Yucatan mini pigs (Sus Scrofa Domestice) were collected (see Table 1). Mini pigs were 26-31 months of age at euthanasia. Tissue handling is described in detail in Supplemental appendix. Alveolar bone samples from $>30$ female Wistar rats ( $>12$ months) and from $>30$ female BALB/c mice ( $>12$ months) were used (Table 1). Animals were purchased from Charles River Laboratories and aged in-house.

\section{Histology}

Aniline blue is used for decalcified bones and cartilage. Young OVX femoral bone was transversally cut to portray the circular patterns of osteons allowing the blue stain to pick up collagen and expose the osteoid matrix. The stains were submerged in 1\% of aniline blue (B8563, sigma) for 1-5 minutes until a dark navy color developed. Slides were then washed in 1\% acetic acid (A38-212, Fisher) for 5 minutes and repeated twice for consistency of stain (Pilawski et al., 2020).

Ploton silver is used to stain decalcified bones and cartilage. Ploton silver staining is a sensitive staining process used to detect nucleolar organizations canalicular systems in murine bone. The stains were submerged in $50 \%$ silver nitrate and 1\% formic, $2 \%$ gelatin in a $2: 1$ ratio, respectfully, for 55 minutes in a dark humidified chamber. Slides were then stained with $5 \%$ sodium thiosulfate.

Picrosirius red (with polarized light, to visualize collagen organization). Slides were stained with $0.1 \%$ sirius red (35780, Pfaltz \& Bauer, CT) dissolved in a saturated picric acid solution for 60 minutes then washed in $1 \%$ acetic acid. Slides were dehydrated in an ascending ethanol series followed by Citrisolv and cover-slipped with Permount. For TRAP staining in human, rat, and mouse tissues, slides were treated with a tartrate resistant acid phosphatase (TRAP) activity kit (386A, Sigma) precisely according to the manufacturer's instructions. For TRAP staining in mini pig tissues, the incubation time was extended from 20-60 minutes to $\sim 3$ hours.

To detect ALP activity in human, mini pig, rat, and mouse tissues, BCIP (Roche, 113832201) and NBT (Roche, 11383213001) were used according to the manufacturer's recommendation, with one exception: in mini pig samples the incubation time was extended from 30 minutes to overnight.

\section{Cellular Assays}

TRAP stains were treated with tartrate-resistant acid phosphatase to pick up the activity of osteoclasts. The instructions were provided by the manufacturer. Tartrate resistant acid phosphatase (TRAP) activities followed published protocols (Yuan et al. 2018). Tartrate-resistant acid phosphatase (TRAP) activity was observed using a leukocyte acid phosphatase staining kit (Sigma). After developing, the slides were dehydrated in a series of ethanol and xylene and subsequently cover-slipped with paramount mounting media.

ALP stains were treated with alkaline phosphatase to detect early osteoblast differentiation. Alkaline phosphatase (ALP) activities followed published protocols (Yuan et al. 2018). Tissue sections were dewaxed and hydrated through a descending grade ethanol series then subjected to histological staining's.

\section{Results}

Histology of aniline blue, ploton silver staining, and picrosirius red; cellular assays including TRAP (tartrate-resistant acid phosphatase) and ALP (alkaline phosphate) were used to analyze murine bone structures and compared to humans, mini pigs, and rats. 

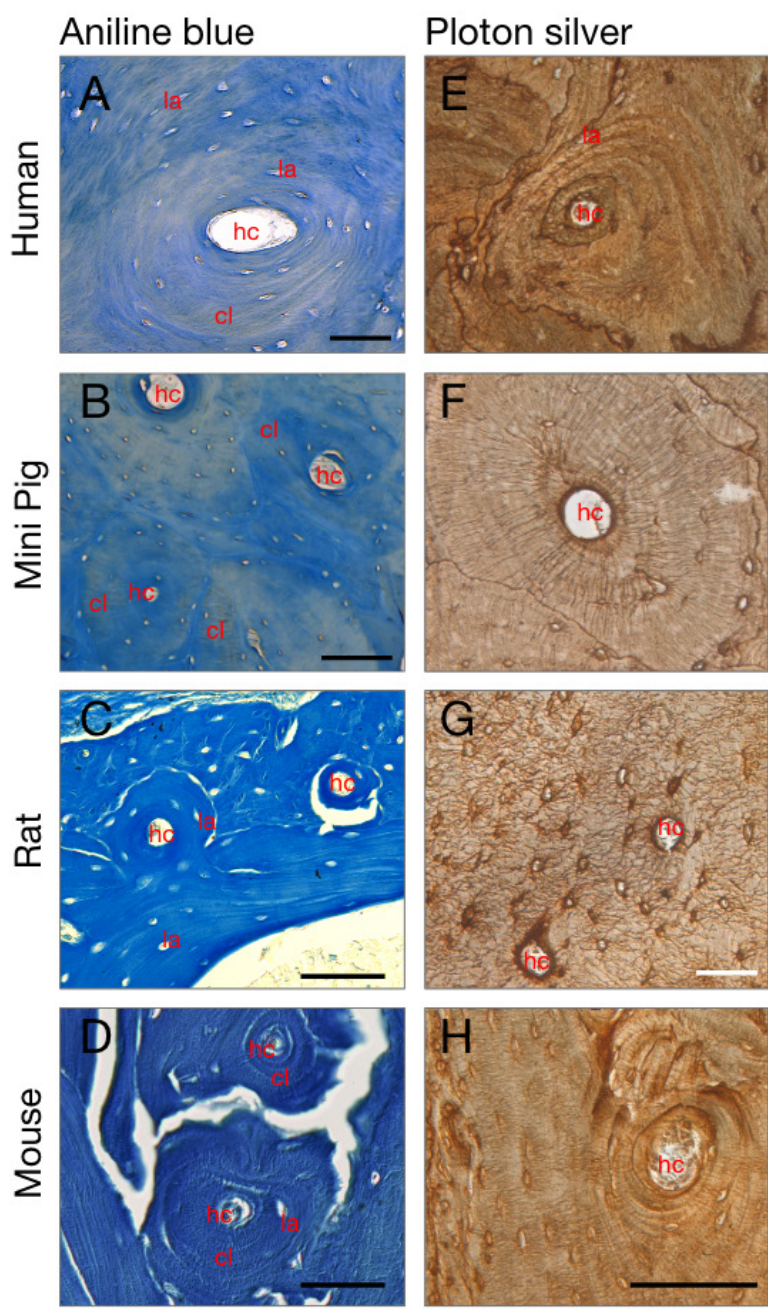
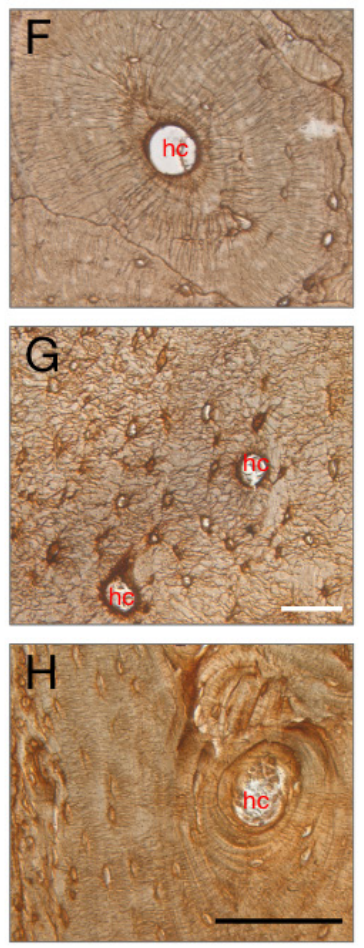
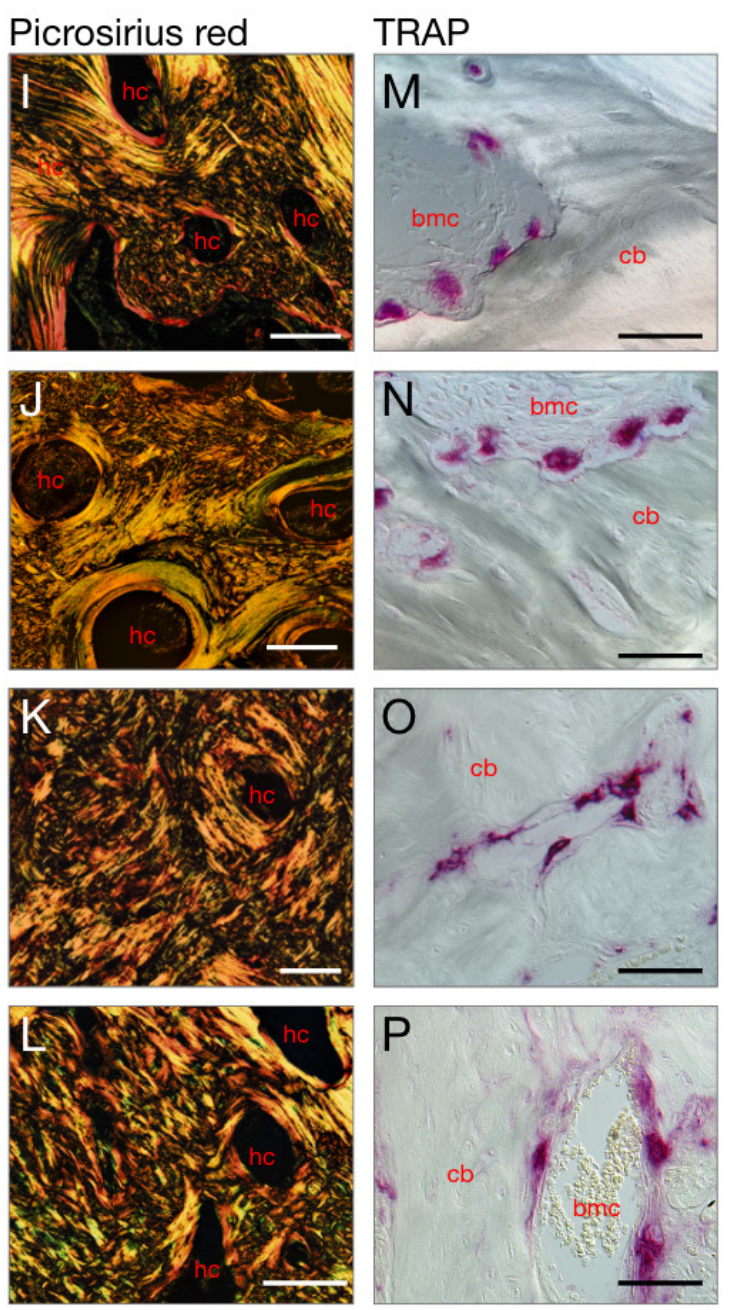
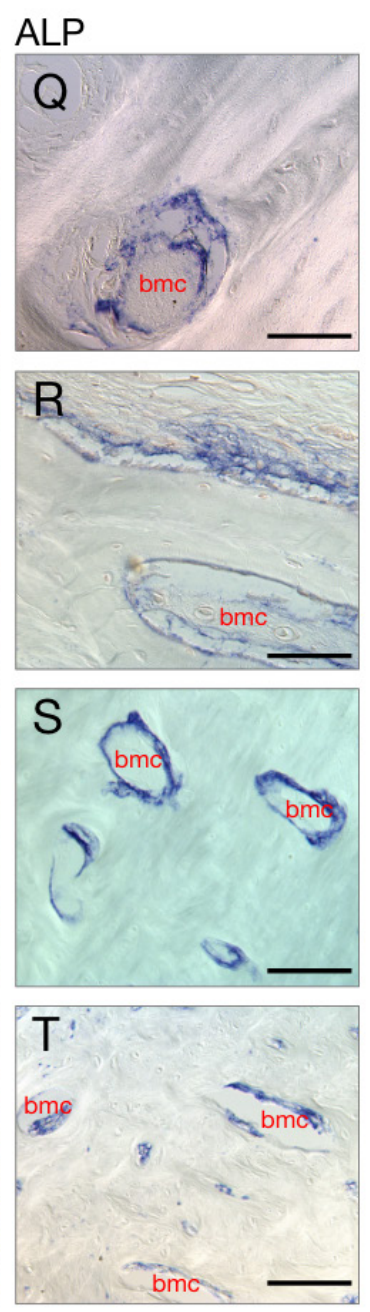

Figure 1. Representative transverse tissues sections through cortical bone stained with aniline blue are shown in (A) human, (B) mini pig, (C) rat, and (D) mouse. Aniline blue staining was used to illustrate collagen organization in human, mini pig, rat, and mouse to demarcate collagen organization. Ploton silver staining was used to demonstrate osteocyte lacunae and canaliculi in (E) human, (F) mini pig, (G) rat, and (H) mouse alveolar bone. Representative transverse tissues sections stained with picrosirius red stained of alveolar bone in (I) human, (J) minipig, (K) rat, and (I) mouse demonstrating cement lines and Haversian canals. Tartrate-resistant acid phosphatase (TRAP) stained of alveolar bone in $(\mathrm{M})$ human, $(\mathrm{N})$ mini pig, $(\mathrm{O})$ rat, and $(\mathrm{P})$ mouse signifies osteoclast activity. Alkaline phosphatase (ALP) stained of alveolar bone in (Q) human, $(\mathrm{R})$ mini pig, $(\mathrm{S})$ rat, and $(\mathrm{T})$ mouse signifies osteoblast activity Abbreviations: hc, Haversian canal; cl, cement lines; la, lacunae; bmc, bone marrow cavity; cb, cortical bone. 


\section{Journal of Student Research}

\section{Aniline Blue}

Aniline blue was used to stain the collagen organization in bone in order to illustrate microstructures including osteons, circumferential bone lamellae, Haversian canals, and osteocyte lacunae. Circumferential lamellae and cement lines were more pronounced in bone from human and mini pig (Fig. 1A, B) than in bone from rat and mouse (Fig. 1C, D). Most mini pig osteocyte lacunae were empty, which most likely reflected inherent difficulties in preserving cell architecture in large animal models (Pilawski et al. 2020). Osteons were clustered together, which was observed in all aniline blue stains (Fig. 1A-D). The white, irregular spacings between the osteons in the rat and mouse tissue samples (Fig. 1C, D) were sectioning artifacts.

\section{Ploton Silver}

Ploton silver is used to stain lacuno-canalicular network in craniofacial tissue to show osteocyte lacunae and canaliculi. Silver ions from the silver nitrate bonded to protein functional groups and caught on to osteocytes. Canaliculi were seen branching out from each lacuna (Fig 1. E-H). In all species under study, ploton silver stains portrayed an intricate network of reticular fibers around osteolytic lacunae. All four species contained an empty lacuna and Haversian canals; the concentric patterns from the cement lines are also seen in all species except for rat.

\section{Picrosirius Red}

Samples of human, mini pig, rat, and mouse alveolar bone (Fig. 1I-L) were stained with picrosirius red to visualize collagen fiber arrangements in bone. The osteonal structures were clustered together with cement lines overlapping one another. Haversian canals were indicated by the dark circles across all species (Fig 1I-L) indicated by "hc" abbreviations. The interwoven arrangement of collagen fiber is known as a basket-weave pattern, which are illustrated by red and orange hues (Adam, 2020). The basket-weave pattern was more observed in rat and mouse samples (Fig $1 \mathrm{~K}, \mathrm{~L}$ ) than in human and mini pig samples (Fig 1I, J). Surrounding the mouse Haversian canals (Fig. 1L), green colored remnants of collagen indicated a thin, irregular arrangements and reticular fibers (Kiernan, 2011). Human and mini pig alveolar samples (Fig. 1I, J) prominently illustrated yellow concentric patterns around the Haversian canals. The yellow hues were representative of tightly aligned and large collagen fibers (Lattouf, 2014).

\section{TRAP}

TRAP staining detects osteoclastic activity illustrated by the magenta-colored staining (Filgueira, 2004). TRAP activity was detected in alveolar bone samples in human, mini pig, rat, and mouse (Fig. 1M-P). TRAP activity in human and mini pig samples (Fig. 1M, N) were most prominent in resorption bays along the edge of the cortical bone surface. Resorption bays are indicated by the ruffled border between cortical bone and bone marrow cavities, which contain high amounts of osteoclast activity (Lindunger, 1990). Rat and mouse samples (Fig. 1O, P) differed because TRAP activity was not prominent in resorption bays but rather sparse around the cortical bone. Mouse and rat samples (Fig. 1P) both showed lacunae around the TRAP activity, however, rat lacunae were not as visible as the mouse lacunae.

\section{V. $A L P$}

ALP activity detects osteoblastic activity illustrated by the navy-blue staining (Lee, 2017). ALP activity was detected in cortical bone in samples from human, mini pig, rat, and mouse (Fig. 1Q-T). ALP activity in all samples along the surfaces was positive around the resorption bays of the bone marrow cavities. 


\section{Discussion}

It was previously assumed that mouse bone physiology and anatomy is fundamentally different from humans (Jilka, 2013). Therefore, researchers rather opted for mini pig tissue. Our results indicate that murine bone does not significantly differ from human, mini pig, and rat bone. Mice, however, are amendable to a wide variety of cellular and immunohistological assays (e.g. Runx2, Rankl) that often do not work in mini pigs. Therefore, it is possible to acquire more information from murine bone resulting in a reduced need of animals. This would adhere to the three $\mathrm{R}$ 's principles aforementioned in the introduction. Our study concluded that bone remodeling and composition in all four species of bone are conserved. Differences in all species were found in age, anatomical location, and whether or not the bone was weight-bearing. These differences, however, had no impact on the remodeling composition, healing (was not examined in this study), and microstructures.

Aniline blue stained all important components of bone tissue, illustrating key microstructures: osteons, circumferential bone lamellae, Haversian canals, and osteocyte lacunae. As indicated by the hc labels, Haversian canals are present in all aniline blue samples (Fig. 1A-D, hc) even though a previous study suggested that mice and rats lack Haversian cortical bone remodeling (Duranova 2014). The identification of osteonal structures was achieved visually by identifying key features such as the Haversian canal surrounded by circumferential bone lamellae. Osteocyte lacunae within the bone matrix were observed across all species (Fig. 1A-D, la). Comparing the presence of osteocytes across all samples has physiological implications because osteocytes contribute to the process of bone remodeling via the activation and regulation of bone resorbing and bone forming cells (Bonewald, 2011). Across all species, osteocytic lacunae were identified as white voids within the bone matrix and indicated the existence of where previous osteocytes existed. Osteocytes were not visible within the lacunae of human and mini pig (Fig 1A, B) due to difficulties in preserving cell architecture in large animals (Pilawski et al. 2020). Nonetheless the morphological similarities are apparent with the presence of Haversian canals and lacunae in all species (Fig. 1A-D).

Ploton silver stain is a sensitive colorimetric method use to render lacuno-canalicular network, a component of bone marrow (Fig. 1E-H) (Fowler et al, 2017). The canaliculi are most concentrated around lacunae in all samples. Reticular fibers lie between the walls of Haversian canals providing structural support, which is why the samples portray a dense network of fibers (Fig 1E-H) (Milovanovic et al, 2015). One study stated that the canalicular structures of mice were disorganized and the bone density was significantly lower than human bone; however, our study showed that Ploton silver staining samples (Fig. 1E-H) contained dense networks in both human and mouse (Moreno-Jimenez et al. 2020). The mouse sample clearly portrayed a tightly bounded lamellar-canalicular system that paralleled the human. All four species show an apparent meshwork of reticular fibers, depicting the morphological similarities in all species. The configurations of the lacuno-canalicular networks depends on the surrounding bone type; for human cortical bone, the canaliculi are co-aligned with the collagen matrix rather than surrounding the Haversian canal (Fig 1E). This is also shown in mice where the lacuno-canicular systems are arranged along the axis of the collagen fibers rather than directed towards the Haversian canals (Fig 1H). The lamellar bone contains flattened lacunae and aligns canaliculi while woven bone contains rounder and irregularly shaped canaliculi (Schemenz et al., 2020); the lacunocanalicular systems in rat are completely irregular depicting the location of woven bone. In mouse however canaliculi tightly align along the collagen matrix and not directed towards the Haversian canals similar to humans depicting the lamellar bone and therefore showing evidence of osteons.

One study found that the rate of remodeling in humans and mouse bone in trabecular bone was the same (Moreno-Jimenez et al. 2020), allowing mice to be viable models for human bone diseases. Magenta stains signified osteoclast activity were shown in all samples (Fig. 1M-P) because TRAP stains for the TRAP enzyme activity. Therefore, osteoclast activity was evidence of bone remodeling and bone growth activity in all samples (Fig 1M-P). Because OVX mice were undergoing maturation at a faster rate than their male counterparts, bone loss and bone 


\section{- Journal of Student Research}

remodeling was detected around bone resorption cavities for all species. Specifically, osteoclastic resorption was occurring at the surface of these cavities. The osteoclasts-indicated in motile cells-move around the bone surface and secrete vesicles with TRAP (Ballanti et al. 1997). The positive TRAP staining comes from a bone surface that was formerly occupied by osteoclasts. The combination of both osteoclast and osteoblast is evidence of bone remodeling. Increased level of ALP refers to increased bone activity. High ALP activity was found in areas of new bone surface as indicated by navy blue around reabsorption bays (Li et al, 2019). Because ALP and TRAP activity was found the around the circumference of the bone marrow cavities, bone remodeling amongst all four species was conserved. The breakdown of bone is the first step to bone remodeling; however, evidence of bone remodeling does not constitute the discovery of osteons, as bone remodeling can occur without forming the characteristic appearance of an osteon.

Samples of human, mini pig, rat, and mouse alveolar bone (Fig. 1I-L) were stained with picrosirius red to visualize collagen fiber arrangements in bone. Picrosirius red stain only picks up on collagen fiber arrangements, so the Haversian canals were stained black under polarized light to reflect the loss of birefringence. Birefringence, determined by polarized light, gives way to different hues and densities of collagen fibers (Coelho, 2018). This is seen in humans and mini pig, but to a lesser degree. The depicted tissue sections clearly demonstrate morphological similarities in osteonal appearance. Because picrosirius red stains for collagen fiber packing and thickness, it is apparent that the osteons in both human and mini pig species (Fig. 1I, J) are within the hard bone. The texture surrounding the Haversian canal clearly indicates a higher percentage of lamellar bone; however, in mice and rat samples there is a higher volume of interwoven bone, known as the irregular arrangement around the Haversian canals this is possible due to age and whether the bone is weight bearing or not. This, however, does not impede on the fact there are clearly depicted osteons in all four species. Morphological textures shown in all samples were indifferent to one another, warranting the use of mice over other species for bone studies.

\section{Conclusion}

Researchers assume that there is not osteonal remodeling in mice; our, however, results indicate the contrary. By illustrating osteons in mice, we are disproving the major argument for not using mice in bone research. Using mice can be more ethical than using mini pigs and rats as there are more functional and well established cellular and immunohistological assays in mice (e.g. for RUNX2 or RANKL), resulting in more information to acquire for bone research in mice in comparison to large animals like mini pigs. This supports the idea that mice should be further implemented in research for human bone diseases.

\section{Acknowledgements}

We would like to thank Igor Pilawski, Serdar Tulu, and most importantly Jill Helms for providing us with an amazing opportunity to work in her lab and being amazing mentors. Thank you Chianna Li for the unconditional support and being a phenomenal guide in the lab.

\section{References}

Ballanti, P., Minisola, S., Pacitti, M. T., Scarnecchia, L., Rosso, R., Mazzuoli, G. F., \& Bonucci, E. (1997). Tartrateresistant acid phosphate activity as osteoclastic marker: sensitivity of cytochemical assessment and serum assay in comparison with standardized osteoclast histomorphometry. Osteoporosis international: a journal established as result of cooperation between the European Foundation for Osteoporosis and the National Osteoporosis Foundation of the USA, 7(1), 39-43. https://doi.org/10.1007/BF01623458 


\section{Journal of Student Research}

Baron, R., Tross, R., \& Vignery, A. (1984). Evidence of sequential remodeling in rat trabecular bone: morphology, dynamic histomorphometry, and changes during skeletal maturation. The Anatomical record, 208(1), 137145. https://doi.org/10.1002/ar.1092080114

Bonewald L. F. (2011). The amazing osteocyte. Journal of bone and mineral research : the official journal of the American Society for Bone and Mineral Research, 26(2), 229-238. https://doi.org/10.1002/jbmr.320

Chen CH, Pei X, Tulu US, Aghvami M, Chen CT, Gaudilliere D, Arioka M, Maghazeh Moghim M, Bahat O, Kolinski $\mathrm{M}$ et al. 2018. A comparative assessment of implant site viability in humans and rats. J Dent Res. 97(4):451459.

Crespi-Abril, A.C., \& Rubilar (2021). Moving forward in the ethical consideration of invertebrates in e experimentation: Beyond the Three R's Principle. Revista de Biología Tropical, 69(S1), 346-357. DOI

10.15517/rbt.v69iSuppl.1.46366

Coelho, P., Souza, M. V., Conceição, L. G., Viloria, M., \& Bedoya, S. (2018). Evaluation of dermal collagen stained with picrosirius red and examined under polarized light microscopy. Anais brasileiros de dermatologia, 93(3), 415-418. https://doi.org/10.1590/abd1806-4841.20187544

Duranova, H., Martiniakova, M., Omelka, R., Grosskopf, B., Bobonova, I., \& Toman, R. (2014). Changes in compact bone microstructure of rats subchronically exposed to cadmium. Acta veterinaria Scandinavica, 56(1), 64. https://doi.org/10.1186/s13028-014-0064-0

Filgueira L. (2004). Fluorescence-based staining for tartrate-resistant acidic phosphatase (TRAP) in osteoclasts combined with other fluorescent dyes and protocols. The journal of histochemistry and cytochemistry : official journal of the Histochemistry Society, 52(3), 411-414. https://doi.org/10.1177/002215540405200312

Fowler, T. W., Acevedo, C., Mazur, C. M., Hall-Glenn, F., Fields, A. J., Bale, H. A., Ritchie, R. O., Lotz, J. C., Vail, T. P., \& Alliston, T. (2017). Glucocorticoid suppression of osteocyte perilacunar remodeling is associated with subchondral bone degeneration in osteonecrosis. Scientific reports, 7, 44618. https://doi.org/10.1038/srep44618

Frost H. M. (1994). Wolff's Law and bone's structural adaptations to mechanical usage: an overview for clinicians. The Angle orthodontist, 64(3), 175-188. https://doi.org/10.1043 /00033219(1994)064<0175:WLABSA $>2.0 . \mathrm{CO} ; 2^{1}$

Haupt M, Kauschke V, Sender J, et al. Bone status of adult female butyrylcholinesterase gene-deficient mice. International Immunopharmacology. 2015 Nov;29(1):208-214. DOI: 10.1016/j.intimp.2015.06.029.

Inada, M., Matsumoto, C., \& Miyaura, C. (2011). Animal models for bone and joint disease. Ovariectomized and orchidectomized animals. Clinical calcium, 21(2), 164-170.

Jilka R. L. (2013). The relevance of mouse models for investigating age-related bone loss in humans. The journals of gerontology. Series A, Biological sciences and medical sciences, 68(10), 1209-1217. https://doi.org/10.1093/gerona/glt046 Jowsey, J. (1966). Studies of Haversian systems in man and some animals. Journal of anatomy, 100(Pt 4), 857.

1 


\section{Tournal of Student Research}

Jorgensen, A. M., Varkey, M., Gorkun, A., Clouse, C., Xu, L., Chou, Z., Murphy, S. V., Molnar, J., Lee, S. J., Yoo, J. J., Soker, S., \& Atala, A. (2020). Bioprinted Skin Recapitulates Normal Collagen Remodeling in FullThickness Wounds. Tissue engineering. Part A, 26(9-10), 512-526. https://doi.org/10.1089/ten.TEA.2019.0319

Jowsey J. (1966). Studies of Haversian systems in man and some animals. Journal of anatomy, 100(Pt 4), 857864.

Kiernan, J. A. (n.d.). Sirius Red for Collagen Staining Protocol. Retrieved March 04, 2021, from https://med.emory.edu/departments/medicine/divisions/cardiology/research/labs/microscopy-inmedicine/_documents/sirius-red-collagen-staining-protocol.pdf

Lattouf R, Younes R, Lutomski D, Naaman N, Godeau G, Senni K, Changotade S. Picrosirius red staining: a useful tool to appraise collagen networks in normal and pathological tissues. J Histochem Cytochem. 2014 Oct;62(10):751-8. doi: 10.1369/0022155414545787. Epub 2014 Jul 14. PMID: 25023614.

Li M, Fu X, Gao H, Ji Y, Li J, Wang Y. Regulation of an osteon-like concentric microgrooved surface on osteogenesis and osteoclastogenesis. Biomaterials. 2019 Sep;216:119269. doi: 10.1016/j.biomaterials.2019.119269. Epub 2019 Jun 17. PMID: 31247479.

Lee, J. M., Kim, M. G., Byun, J. H., Kim, G. C., Ro, J. H., Hwang, D. S., Choi, B. B., Park, G. C., \& Kim, U. K. (2017). The effect of biomechanical stimulation on osteoblast differentiation of human jaw periosteumderived stem cells. Maxillofacial plastic and reconstructive surgery, 39(1), 7. https://doi.org/10.1186/s40902-017-0104-6

Lindunger, A., MacKay, C. A., Ek-Rylander, B., Andersson, G., \& Marks, S. C., Jr (1990). Histochemistry and biochemistry of tartrate-resistant acid phosphatase (TRAP) and tartrate-resistant acid adenosine triphosphatase (TrATPase) in bone, bone marrow and spleen: implications for osteoclast ontogeny. Bone and mineral, 10(2), 109-119. https://doi.org/10.1016/0169-6009(90)90086-u

Minematsu, A., Yoshimura, O., Yotsuji, H., Ichigo, H., Kobayashi, R., Sasaki, H., Tanaka, S., Kanemura, N., Shirahama, K., \& Miyamoto, H. (2001). Time course of influence by ovariectomy and calcium diet on bone properties in mice. Journal of the Japanese Physical Therapy Association = Rigaku ryoho, 4(1), 19-23. https://doi.org/10.1298/jjpta.4.19

Ott S. M. (2018). Cortical or Trabecular Bone: What's the Difference?. American journal of nephrology, 47(6), 373375. https://doi.org/10.1159/000489672

Parfitt A. M. (1994). Osteonal and hemi-osteonal remodeling: the spatial and temporal framework for signal traffic in adult human bone. Journal of cellular biochemistry, 55(3), 273-286. https://doi.org/10.1002/jcb.240550303

Pereira, A. F., Javaheri, B., Pitsillides, A. A., \& Shefelbine, S. J. (2015). Predicting cortical bone adaptation to axial loading in the mouse tibia. Journal of the Royal Society, Interface, 12(110), 0590. https://doi.org/10.1098/rsif.2015.0590

Pilawski, I., Tulu, U. S., Ticha, P., Schüpbach, P., Traxler, H., Xu, Q., Pan, J., Coyac, B. R., Yuan, X., Tian, Y., Liu, Y., Chen, J., Erdogan, Y., Arioka, M., Armaro, M., Wu, M., Brunski, J. B., \& Helms, J. A. (2020). 


\section{Journal of Student Research}

Interspecies Comparison of Alveolar Bone Biology, Part I: Morphology and Physiology of Pristine Bone. JDR Clinical \& Translational Research. https://doi.org/10.1177/2380084420936979

Schaffler, M. B., \& Kennedy, O. D. (2012). Osteocyte signaling in bone. Current osteoporosis reports, 10(2), 118125. https://doi.org/10.1007/s11914-012-0105-4

Schemenz, V., Gjardy, A., Chamasemani, F. F., Roschger, A., Roschger, P., Zaslansky, P., Helfen, L., Burghammer, M., Fratzl, P., Weinkamer, R., Brunner, R., Willie, B. M., \& Wagermaier, W. (2020). Heterogeneity of the osteocyte lacuno-canalicular network architecture and material characteristics across different tissue types in healing bone. Journal of structural biology, 212(2), 107616. https://doi.org/10.1016/j.jsb.2020.107616

Skedros, J. G., Holmes, J. L., Vajda, E. G., \& Bloebaum, R. D. (2005). Cement lines of secondary osteons in human bone are not mineral-deficient: new data in a historical perspective. The anatomical record. Part A, Discoveries in molecular, cellular, and evolutionary biology, 286(1), 781-803. https://doi.org/10.1002/ar.a.20214

Sözen, T., Özışık, L., \& Başaran, N. Ç. (2017). An overview and management of osteoporosis. European journal of rheumatology, 4(1), 46-56. https://doi.org/10.5152/eurjrheum.2016.048

Turner, R. T., Maran, A., Lotinun, S., Hefferan, T., Evans, G. L., Zhang, M., \& Sibonga, J. D. (2001). Animal models for osteoporosis. Reviews in endocrine \& metabolic disorders, 2(1), 117-127. https://doi.org/10.1023/a:1010067326811

Yuan, X., Pei, X., Zhao, Y., Tulu, U. S., Liu, B., \& Helms, J. A. (2018). A Wnt-Responsive PDL Population Effectuates Extraction Socket Healing. Journal of dental research, 97(7), 803-809. https://doi.org/10.1177/0022034518755719 